cohort had significantly longer PFS (hazard ratio $[\mathrm{HR}]=0.43$, $95 \%$ CI $0.32-0.58, \mathrm{P}<0.0001)$ and significantly longer OS $(\mathrm{HR}=0.43,95 \%$ CI $0.32-0.60, \mathrm{P}<0.0001)$. Furthermore, the simulated-broader cohort demonstrated a survival curve above the simulated-PRIMA curve. Within the 2010-2015 diagnosis (contemporary) stage III cohort $(n=169), 57.4 \%$ had IDS and 42.6\% had PDS, of whom $23.1 \%$ had PDS VRD, $17.2 \%$ had PDS NVRD, and 2.4\% were PDS not evaluable for residual disease.

Conclusion The simulated-broader cohort showed longer duration of OS and PFS outcomes as the survival curves lie above the simulated-PRIMA cohort. This difference is driven by the better prognosis for patients with stage III NVRD after PDS population; this population accounted for approximately $17 \%$ of the contemporary patient cohort at this UK centre.

Disclosures This study was funded by GlaxoSmithKline.

Clinical Trial Registration: N/A

Dr. Hollis reports institutional grants and personal fees from GlaxoSmithKline.

Dr. Gourley reports personal fees from Roche, Clovis, Tesaro, Foundation One, Nucana, Aprea, Novartis, Chugai, and MSD; institutional grants from Clovis, Tesaro, Nucana, and Novartis.

Drs. Kiss, Roebuck, Heffernan and Starkie-Camejo are employees of GlaxoSmithKline.

\section{SYSTEMATIC LITERATURE REVIEW OF EFFICACY AND SAFETY OF FIRST-LINE MAINTENANCE THERAPY TRIALS IN ADVANCED OVARIAN CANCER}

${ }^{1}$ Holly Guy, ${ }^{2}$ Karin Travers, ${ }^{2}$ Carol Hawkes, ${ }^{1}$ Lydia Walder, ${ }^{2}$ Izabela Malinowska,

${ }^{2}$ Divya Gupta. ${ }^{1}$ Fiecon; ${ }^{2}$ Glaxosmithkline

\subsection{6/ijgc-2020-ESG0.127}

Introduction/Background Maintenance therapies have changed the treatment landscape in ovarian cancer (OC) in recent years. Here we sought to review safety and efficacy outcomes in clinical trials of first-line maintenance therapies.

Methodology A systematic literature review (SLR) was performed on 27 February 2020 to identify clinical outcomes associated with first-line maintenance therapies and maintenance therapies initiated alongside first-line chemotherapy followed by a maintenance phase for advanced OC. Randomised controlled trials (RCTs), non-RCTs and observational studies were eligible. Selection criteria following the PICOS (population, interventions, comparators, outcomes and study type) principle were specified. Outcomes of interest were progression-free survival (PFS), overall survival (OS), and treatment emergent adverse events (TEAEs). Selected studies were then extracted by one reviewer and assessed for quality by a second reviewer. Disagreements were resolved by a third reviewer when required.

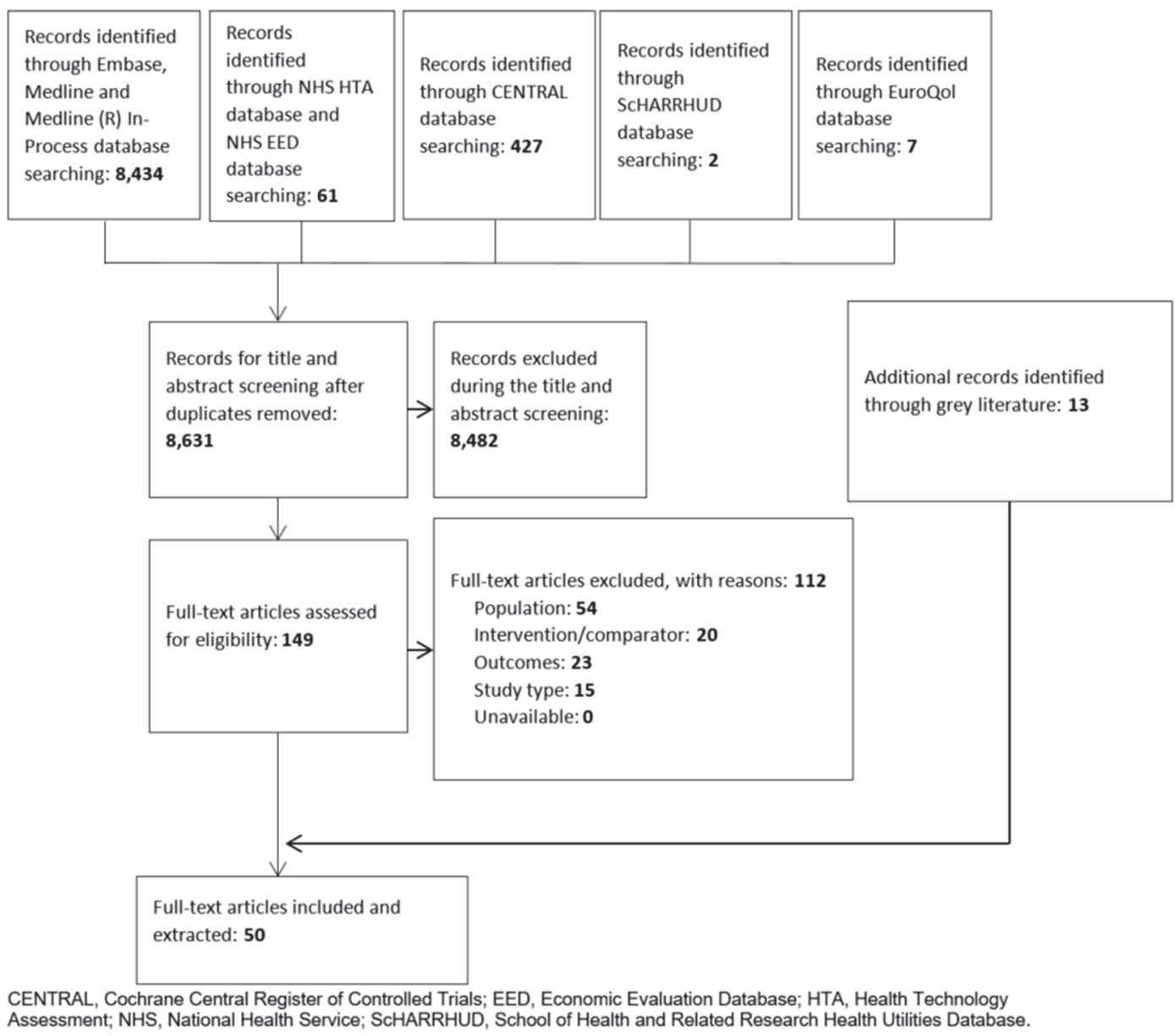

Abstract 373 Figure 1 PRISMA diagram 
Results This SLR retrieved 8,631 unique references of which a total of 50 references were accepted and extracted in this SLR (figure 1).

The 50 references identified covered 18 clinical trials that evaluated maintenance therapies in OC patients following one prior line of chemotherapy. Of these 18 trials, 12 were RCTs and the remaining 6 were observational, dose escalation and retrospective review studies.

Of the 18 trials, only 2 did not assess PFS as an efficacy endpoint (NCT00058435 and MIMOSA). PARP inhibitors across the board reported a better PFS hazard ratio (HR) than other OC maintenance therapies (table 1). No pattern was identified in relation to PFS amongst patients who were treated with a maintenance therapy following first-line platinum-based chemotherapy versus those who received a

Abstract 373 Table 1 PFS and OS studies included in the
analysis

\begin{tabular}{|c|c|c|c|}
\hline Trial & Drug(s) & PFS, HR (95\% Cl) & OS, HR $(95 \% \mathrm{Cl})$ \\
\hline \begin{tabular}{|c|} 
NCT00058435 \\
Sabbatini 2006 \\
\end{tabular} & Abagovomab & NA & NA \\
\hline $\begin{array}{l}\text { MIMOSA } \\
\text { (NCT00418574) } \\
\text { Sabbatini 2013 }\end{array}$ & $\begin{array}{l}\text { Arm 1: Abagovomab } \\
\text { Arm 2: Placebo }\end{array}$ & $1.099(0.919-1.315)^{*}$ & $\begin{array}{l}1.15(0.872- \\
1.1518)\end{array}$ \\
\hline $\begin{array}{l}\text { ICON-7 } \\
\text { (NCT00483782) } \\
\text { Oza 2015 }\end{array}$ & $\begin{array}{l}\text { Arm 1: Chemotherapy + } \\
\text { surveillance } \\
\text { Arm 2: Chemotherapy + } \\
\text { Bevacizumab }\end{array}$ & $0.93(0.83-1.05)$ & $0.99(0.85-1.14)$ \\
\hline \begin{tabular}{|c|} 
AGO-OVAR16 \\
(NCT00866697) \\
Du Bois 2014 \\
\end{tabular} & $\begin{array}{l}\text { Arm 1: Pazopanib } \\
\text { Arm 2: Placebo }\end{array}$ & $0.766(0.643-0.911)$ & $\begin{array}{l}0.96(0.805- \\
1.145)^{\dagger}\end{array}$ \\
\hline \begin{tabular}{|l} 
SOLO-1 \\
(NCT01844986) \\
Moore 2018 \\
\end{tabular} & $\begin{array}{l}\text { Arm 1: Olaparib } \\
\text { Arm 2: Placebo }\end{array}$ & $0.30(0.23-0.41)$ & $0.95(0.60-1.53)$ \\
\hline \begin{tabular}{|l|} 
Knipprath-Meszaros \\
2017 \\
\end{tabular} & $\begin{array}{l}\text { Arm 1: Letrozole } \\
\text { Arm 2: "Do Nothing" }\end{array}$ & NR & NA \\
\hline Goel 2017 & $\begin{array}{l}\text { Arm 1: Tamoxifen } \\
\text { Arm 2: Treatment } \\
\text { Surveillance }\end{array}$ & NR & NA \\
\hline \begin{tabular}{|l|} 
AGO-OVAR16 \\
(NCT00866697); \\
NCT01227928 (East \\
Asian sub study) \\
Kim 2018
\end{tabular} & $\begin{array}{l}\text { Arm 1: Pazopanib } \\
\text { Arm 2: Placebo }\end{array}$ & $1.114(0.818-1.518)$ & NR \\
\hline \begin{tabular}{|c|} 
NCT01227928 \\
Zang 2013 \\
\end{tabular} & $\begin{array}{l}\text { Arm 1: Pazopanib } \\
\text { Arm 2: Placebo }\end{array}$ & $0.98(0.593-1.620)$ & NA \\
\hline \begin{tabular}{|l} 
JGOG3022 \\
(NCT00951496) \\
Komiyama 2019 \\
\end{tabular} & $\begin{array}{l}\text { Arm 1: Chemotherapy + } \\
\text { Bevacizumab }\end{array}$ & NR & NA \\
\hline $\begin{array}{l}\text { OSCAR } \\
\text { (NCT01863693) } \\
\text { Hall 2018 }\end{array}$ & $\begin{array}{l}\text { Arm 1: Chemotherapy + } \\
\text { Bevacizumab + } \\
\text { Bevacizumab } \\
\text { maintenance }\end{array}$ & NR & NA \\
\hline $\begin{array}{l}\text { PAOLA-1 } \\
\text { (NCT02477644) } \\
\text { Ray-Coquard 2019 }\end{array}$ & $\begin{array}{l}\text { Arm 1: Olaparib + } \\
\text { Bevacizumab } \\
\text { Arm 2: Placebo + } \\
\text { Bevacizumab }\end{array}$ & $0.59(0.49-0.72)$ & NR \\
\hline $\begin{array}{l}\text { PRIMA } \\
\text { (NCT02655015) } \\
\text { Gonzalez-Martin } 2019\end{array}$ & $\begin{array}{l}\text { Arm 1: Niraparib } \\
\text { Arm 2: Placebo }\end{array}$ & $0.62(0.5-0.76)$ & $0.7(0.44-1.11)$ \\
\hline $\begin{array}{l}\text { CHIVA/GINECO } \\
\text { (NCT01583322) } \\
\text { Feron 2019 }\end{array}$ & \begin{tabular}{|l|} 
Arm 1: NACT + \\
Nintendanib + \\
Nintendanib \\
maintenance \\
Arm 2: NACT + Placebo \\
+ Placebo maintenance \\
\end{tabular} & 1.5 & 1.54 \\
\hline $\begin{array}{l}\text { ESME (NCT03275298) } \\
\text { Romeo } 2019\end{array}$ & $\begin{array}{l}\text { Arm 1: Platinum-based } \\
\text { Chemotherapy + } \\
\text { maintenance }\end{array}$ & NR & NR \\
\hline \begin{tabular}{|l} 
TRINOVA-3 \\
(NCT01493505) \\
Vergote 2019
\end{tabular} & $\begin{array}{l}\text { Arm 1: Chemotherapy + } \\
\text { Trebananib + Trebananib } \\
\text { maintenance } \\
\text { Arm 2: Chemotherapy + } \\
\text { Placebo + Placebo } \\
\text { maintenance }\end{array}$ & $0.93(0.79-1.09)$ & $0.99(0.79-1.25)$ \\
\hline $\begin{array}{l}\text { VELIA/GOG-3005 } \\
\text { (NCT02470585) } \\
\text { Coleman 2020 }\end{array}$ & $\begin{array}{l}\text { Arm 1: Chemotherapy + } \\
\text { Veliparib + Veliparib } \\
\text { maintenance } \\
\text { Arm 2: Chemotherapy + } \\
\text { Placebo + Placebo } \\
\text { maintenance }\end{array}$ & $0.68(0.56-0.83)$ & NR \\
\hline $\begin{array}{l}\text { AGO-OVAR12 } \\
\text { (NCT01015118) } \\
\text { Ray-Coquard 2017 }\end{array}$ & $\begin{array}{l}\text { Arm 1: Chemotherapy + } \\
\text { Nintendanib + } \\
\text { Nintendanib } \\
\text { maintenance } \\
\text { Arm 2: Chemotherapy + } \\
\text { Placebo + Placebo } \\
\text { maintenance }\end{array}$ & NR & $0.99(0.83-1.17)$ \\
\hline $\begin{array}{l}\text { GOG-0218 } \\
\text { (NCT00262847) } \\
\text { Burger 2011 }\end{array}$ & $\begin{array}{l}\text { Arm 1: Chemotherapy + } \\
\text { Bevacizumab + } \\
\text { Bevacizumab } \\
\text { maintenance } \\
\text { Arm 2: Chemotherapy + } \\
\text { Placebo + Placebo } \\
\text { maintenance }\end{array}$ & $0.77(0.681-0.870)$ & $0.915^{\ddagger}$ \\
\hline
\end{tabular}

maintenance drug concurrently with first-line platinum-based chemotherapy and then continued with the maintenance treatment.

OS was reported as a secondary endpoint in 12 trials (MIMOSA, AGO-OVAR16, SOLO-1, ICON-7, GOG-0218, AGO-OVAR12, VELIA/GOG-3005, TRINOVA-3, ESME, CHIVA/GINESCO, PRIMA and PAOLA-1). Only PARP inhibitor-containing therapies reported significant OS HRs below 1 across all trial populations.

TEAEs were reported for 11 of the 18 trials. Discontinuation due to AEs was reported in 10 of the 18 trials.

Conclusion Therapies that included PARP inhibitors reported better PFS HR than other OC maintenance therapies. In study populations including both BRCA mutation positive and wild type, clinical benefit is conferred by both olaparib plus bevacizumab and niraparib as indicated by PFS. OS data remain immature.

Disclosures This study was funded by GlaxoSmithKline.

Clinical Trial Registration: N/A

Dr Guy and Walder report institutional reimbursements from GlaxoSmithKline.

Drs. Travers, Hawkes, Malinowska, and Gupta are employees of GlaxoSmithKline.

\section{REAL WORLD PROGNOSTIC RELEVANCE OF RESIDUAL DISEASE AND OTHER CLINICAL FACTORS ON THE PROGRESSION OF DISEASE AND DEATH IN PATIENTS WITH ADVANCED OVARIAN CANCER IN THE US}

${ }^{1}$ Dana Chase, ${ }^{2}$ Jessica Perhanidis, ${ }^{2}$ Divya Gupta, ${ }^{3}$ Linda Kalilani, ${ }^{2}$ Leah Sansbury, ${ }^{2}$ Tatia Woodward, ${ }^{3}$ Antonio González-Martín. ${ }^{1}$ University of Arizona Cancer Center: ${ }^{2}$ Glaxosmithkline; ${ }^{3}$ Grupo Español de Investigación en Cáncer de Ovario (GEICO) and the Medical Oncology Department, Clínica Universidad de Navarra, Madrid, Spain

\subsection{6/ijgc-2020-ESGO.128}

Introduction/Background Although most patients with ovarian cancer (OC) respond to first line (1L) treatment, 70\% of women experience disease progression (PD) within 3 years. Identifying prognostic factors that impact survival is crucial to identify patients who may benefit from new treatment regimens such as maintenance therapies. The objective of this study was to assess the association between visible residual disease (VRD) following interval (IDS) or primary debulking surgery (PDS) and other clinical factors, and the risk of PD or death in patients with advanced OC in a real-world setting. Methodology This retrospective cohort study included patients diagnosed with invasive ovarian cancer between January 1, 2011 and February 29, 2020, from the Flatiron Health electronic health record-derived de-identified US database (most OC patients $(87 \%)$ originate from community oncology practices). Inclusion/exclusion criteria are shown in table 1 . The index date (ID) was defined as the last date of $1 \mathrm{~L}$ treatment. Multivariate Cox regression models were used to identify demographic and clinical factors associated with time to next

Abstract 374 Table 1 Inclusion/Exclusion criteria

\begin{tabular}{|l|l|}
\hline Inclusion criteria & Exclusion criteria \\
\hline Patients were $\geq 18$ years old & Missing an IDS or PDS date \\
\hline Stage III or IV disease & Had IDS or PDS and unknown VRD status \\
\hline Received any 1L treatment & \\
\hline $\begin{array}{l}\text { Had } \geq 12 \text { weeks of follow-up after completing 1L } \\
\text { treatment }\end{array}$ & \\
\hline
\end{tabular}

